\title{
KELVIN-HELMHOLTZ INSTABILITIES AND THE EMISSION KNOTS IN HERBIG-HARO JETS
}

\author{
S. MASSAGLIA, M. MICONO AND A. FERRARI \\ Dipartimento di Fisica Generale dell'Università, \\ Via Pietro Giuria 1, I-10125 Torino, Italy \\ AND \\ G. BODO AND P. ROSSI \\ Osservatorio Astronomico di Torino, \\ Strada dell'Osservatorio 20, I-10025 Pino Torinese, Italy
}

\begin{abstract}
We discuss the non-linear evolution of Kelvin-Helmholtz instabilities in Herbig-Haro jets performing numerical simulations by means of a PPM hydro-code modified as to include non-equilibrium, optically thin, radiation losses and heating. In this paper we discuss in particular the effects of different functional dependences of heating on density. The results obtained show a weak dependency of the instability evolution on the different forms of the heating function, that is largely unknown, therefore the simple assumption of constant heating, adopted in previous papers on this matter, does not lead to severe limitations on the general applicability of the results to the astrophysical jets and, in particular, to the origin of the emission knots.
\end{abstract}

\section{Introduction}

Recent wide field-of-view observations of Herbig-Haro jets (Devine 1997) have revealed that they extend for more than a few parsecs and, therefore, they last more than $10^{5} \mathrm{yr}$. This implies that these jets successfully survive dynamical instabilities, the most relevant of them being the KelvinHelmholtz instability. Moreover, many $\mathrm{HH}$ jets show chains of quasi-periodically spaced, shock excited, knots (see e.g. Reipurth and Heathcote 1992; Eislöffel and Mundt 1992; Reipurth, Raga and Heathcote 1992) that emit mainly in $\mathrm{H}_{\alpha}$, [SII] and [OIII] optical lines. 
The origin of these emission knots is controversial. It has been suggested (Raga et al. 1990) that sudden variations in the mass ejection velocity from the central source, with a repetition time scale of the order of a hundred years, may give rise to working surfaces, internal to the jet, that travel as shocks at a speed very close to the jet's velocity. Alternatively, Bührke, Mundt and Ray (1988) proposed that Kelvin-Helmholtz instabilities themselves may be responsible for the knot formation, since $\mathrm{K}-\mathrm{H}$ reflected modes can grow up to non-linear amplitudes leading to biconical shocks aligned along the jet's longitudinal axis.

Studies of the stability of jets against K-H instabilities has been carried out by several authors both in the linear (see Birkinshaw 1991 and references therein) and non-linear (e.g. Bodo et al. 1994 and 1995, Norman and Hardee 1988) regimes. In applying the K-H instability mechanism to the case of $\mathrm{HH}$ jets, a basic physical process must be included in the picture: radiative losses. These have been considered, in the linear limit, by Massaglia et al. (1992) who found that radiative losses tend to stabilize K-H reflected modes. Massaglia et al. (1992) adopted the Raymond-Smith (1977) cooling function with a constant heating, i.e. independent of the physical parameters. More recently, Hardee and Stone (1997) have analyzed the linear stability of a slab jet for different power-law cooling functions and heating independent of temperature and proportional to the density: they find that the growth of $\mathrm{K}-\mathrm{H}$ modes is reduced for a cooling that is a steep function of temperature, while it is enhanced for a cooling that is a shallow function of temperature.

Besides linear studies of radiative K-H instabilities, the non-linear evolution has been also explored in recent years. Several authors (see Massaglia et al. 1996; Rossi et al. 1997; Micono et al. 1997; Stone, Xu and Hardee 1997) have tackled this problem by means of numerical hydro-codes. In general, they found that radiative losses play a significant role in the instability evolution which is crucially dependent on the assumed form of the cooling function assumed.

The instability evolution may, in general, depend crucially on the amount and density dependence of the heating term as well. The problem is that local heating is not an observable quantity and we can only argue about the relative importance of the various physical processes (ionizing radiation from the central star, turbulence, resistive dissipation of magnetic fields, etc.) that can possibly contribute to the energy input.

In this paper we analyze the spatial non-linear growth of K-H axisymmetric modes for a cylindrical jet in presence of non-equilibrium radiative losses and consider two kind of heating terms: i) a constant heating, and ii) a heating proportional to the particle density. Both kinds of heating are normalized to initially balance radiative losses. The plan of the paper is the 
following: in Section 2 we present the physical problem, the results obtained are discussed in Section 3 and the conclusions are drawn in Section 4.

\section{The Physical Problem}

We have carried out a spatial perturbation analysis by solving the hydrodynamic equations for the mass and momentum conservation for a 2-D cylindrical fluid jet, employing a two-dimensional PPM code (Colella and Woodward 1984, Bodo et al. 1995), modified by means of an operator splitting technique (see Rossi et al. 1997) to include radiative losses and heating in the energy equation. We refer to Massaglia et al. (1996) and Rossi et al. (1997) for the details of the radiation treatment and to Micono et al. (1997) for the characteristics of the initial configuration, perturbation, boundary conditions and the integration domain. The energy equation is written as

$$
\frac{\partial E}{\partial t}+\nabla \cdot(E \vec{v})=-p(\nabla \cdot \vec{v})+(\mathcal{H}-\mathcal{L}),
$$

where the fluid variables $p, \vec{v}$ and $E$ are, as customary, the pressure, velocity, and thermal energy respectively; $\mathcal{H}$ and $\mathcal{L}$ represent the energy input and loss term (energy per unit volume per unit time) which includes energy lost in lines and in the ionization and recombination processes. In the equilibrium configuration, at $t=0$, we have:

$$
\mathcal{H}_{0}-\mathcal{L}_{0}=0 \text {. }
$$

Our goal is to study the effects of different assumptions for the heating term, we have therefore carried out calculations setting first $\mathcal{H}=$ constant and then $\mathcal{H}$ proportional to the particle density $n_{\mathrm{H}}$. We remind that the evolution time is measured in units of $t_{\mathrm{cr}}=a / c_{\mathrm{S}}\left(c_{\mathrm{s}}\right.$ is the isothermal sound speed) and the velocities in units of $c_{\mathrm{s}}$.

We recall that the parameters that control the dynamics of the system are the jet Mach number $M$, and the infinity to on-axis density ratio $\nu \equiv$ $n_{\infty} / n_{0}$. The radiation effects instead are fully determined setting the initial on-axis temperature $T_{0}$ and the cooling time $\tau_{\text {rad }} \equiv t_{\text {rad }} / t_{\mathrm{cr}}$, with $t_{\mathrm{rad}}=$ $p /[(\Gamma-1) \mathcal{L}]$, or equivalently the column density $n_{0} a$. As far as the heating time scale $\tau_{\text {heat }}$ is concerned, we note that, this parameter being initially set equal to $\tau_{\text {rad }}$, it is not an independent parameter. As discussed in Rossi et al. (1997), the initial parameters appropriate for the application to stellar jets yield $\tau_{\text {rad }} \gg 1$, i.e. the initial conditions are nearly adiabatic. As unstable modes grow and shocks form, radiation effects become important locally in the compressed regions.

In the calculation we have set $M=10, \nu=1, T_{0}=10,000 \mathrm{~K}$ and $n_{0} a=$ $10^{18} \mathrm{~cm}^{-2}$, which implies a jet mass flux $\approx 10^{-10} \times a_{15} M \mathrm{M}_{\odot} \mathrm{yr}^{-1}$ and a momentum flux $\approx 10^{-9} \times a_{15} M^{2} \mathrm{M}_{\odot} \mathrm{km} \mathrm{s}^{-1} \mathrm{yr}^{-1}$, with $a_{15}=a /\left(10^{15} \mathrm{~cm}\right)$. 
Constant heating

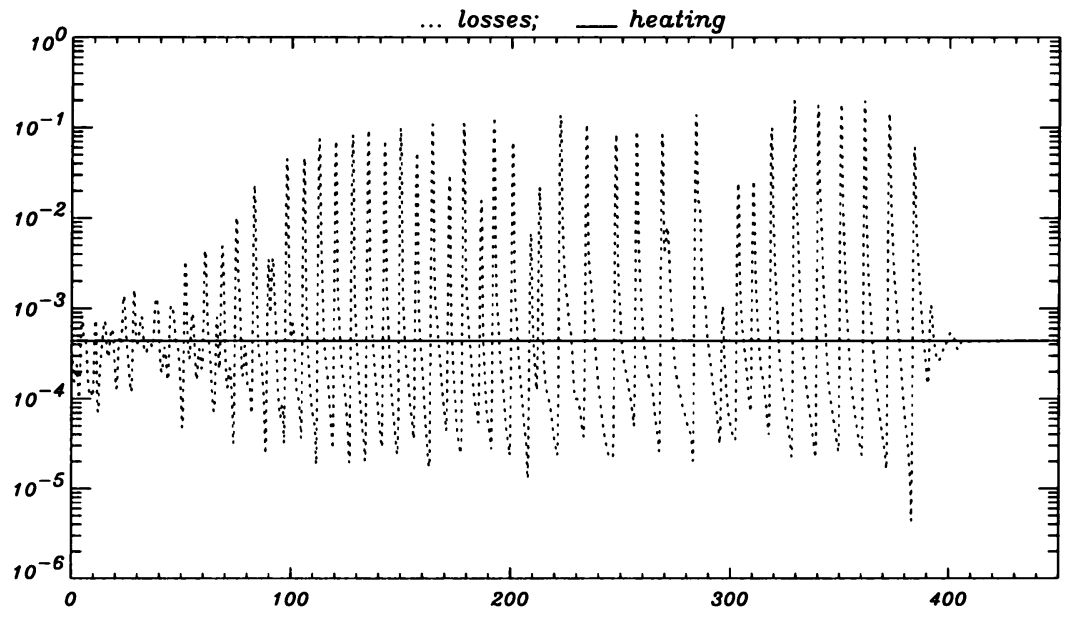

Heating proportional to density

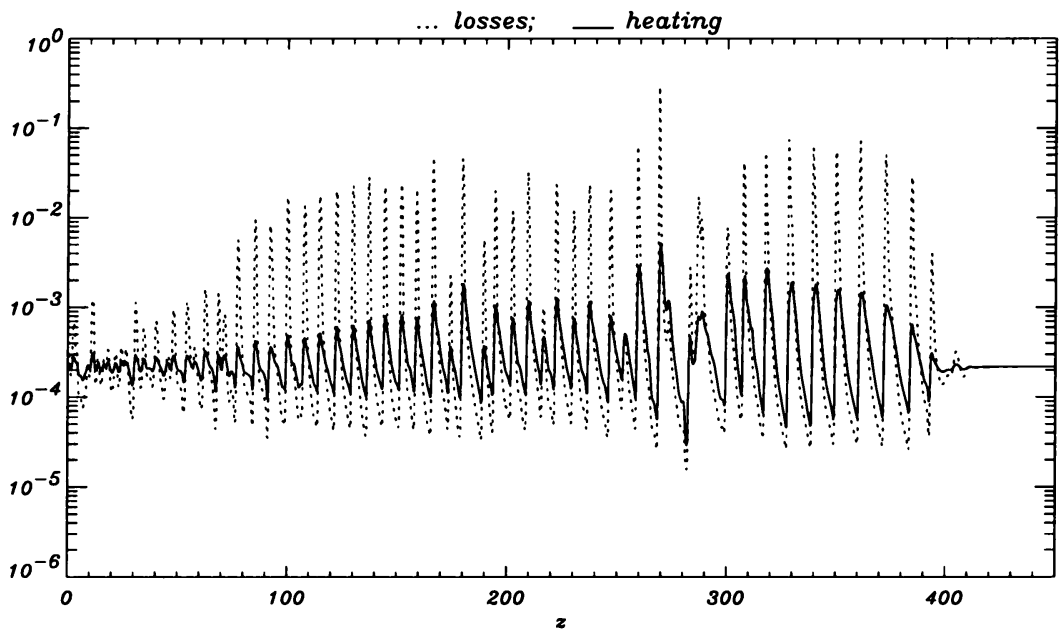

Figure 1. On-axis behavior of radiative losses (dashed line) and heating (solid line) for $\mathcal{H}=$ constant (upper panel) and for $\mathcal{H} \propto n_{\mathrm{H}}$ (lower panel) against $z$. Figure refers to the evolution time $\tau=32$ 
In Figure 1 we plot, at the evolution time $\tau=32$ and in arbitrary units, the on-axis behavior of radiative losses (dashed line) and heating (solid line) vs the longitudinal distance $z$, measured in units of $a$, for $\mathcal{H}=$ constant (upper panel) and for $\mathcal{H} \propto n_{\mathrm{H}}$ (lower panel). We note that a non-constant heating tends to reduce the amplitude of losses in shocks, therefore one can expect for this case radiation effects slightly reduced, i.e. larger shock temperatures and a little more vigorous mixing (see Rossi et al. 1997). Figure 2 is a gray-scale image of the logarithmic density distribution where we show a section of the jet going from 150 up to 400 radii and the two panels are again snapshots of the evolution as in Figure 1. We note that the two morphologies look very much qualitatively alike, albeit they present differences in the details, as shown in the magnified frames: in the nonconstant heating case (bottom panel) we notice a tendency to form wider shocks, e.g. the formation at $\sim 260$ radii, and to send material and acoustic waves into the ambient medium in a more vigorous way.

An important tool for capturing the dynamical behavior of the system is the average momentum carried along by the jet. In Figure 3 we plot, at $\tau=32$ (top panels) and $\tau=38.5$ (bottom panels), the momentum carried out by the jet particles as a function of $z$ and averaged over slices transversal to the jet axis and of longitudinal width of 50 grid points. We show, from left to right, the behavior in the adiabatic limit, the case with $\mathcal{H}=$ constant and the case with $\mathcal{H} \propto n_{\mathrm{H}}$. Examining this figure we notice that, in the adiabatic case, the jet loses almost entirely its momentum at distances $\gtrsim 230 a$ due to instability-driven mixing with the ambient medium (see Bodo et al. 1994 and 1995). Radiation losses reduce sensibly the mixing behavior of the jet, with respect to the adiabatic case, since cooling limits the strength of the shocks that tend to inflate the jet (see discussion in Rossi et al. 1997 and Micono et al. 1997). Looking at the differences between the two different assumed $\mathcal{H}$ we see that they, qualitatively, behave in the same way, apart from some details such as a larger increase in the jet momentum at $\tau=38.5$ and for $\mathcal{H} \propto n_{\mathrm{H}}$, at $z \sim 250 a$, just before the mixing develops.

\section{Conclusions}

We have studied the non-linear spatial evolution of axisymmetric modes in a cylindrical jet in presence of radiative losses and two different heating terms: i) $\mathcal{H}=$ constant and ii) $\mathcal{H} \propto n_{\mathrm{H}}$. The numerical results show that the general evolution does not depend crucially on the form of $\mathcal{H}$, even though the details may do so. The physical reason for this is that the initial cooling time $\tau_{\text {rad }}$ is much larger than unity, and so is the heating time $\tau_{\text {heat }}$; as shocks form, losses grow as $\propto n_{\mathrm{H}}^{2}$ in the compression regions downstream, while heating, at most, grows as $\propto n_{\mathrm{H}}$. In the decompression 

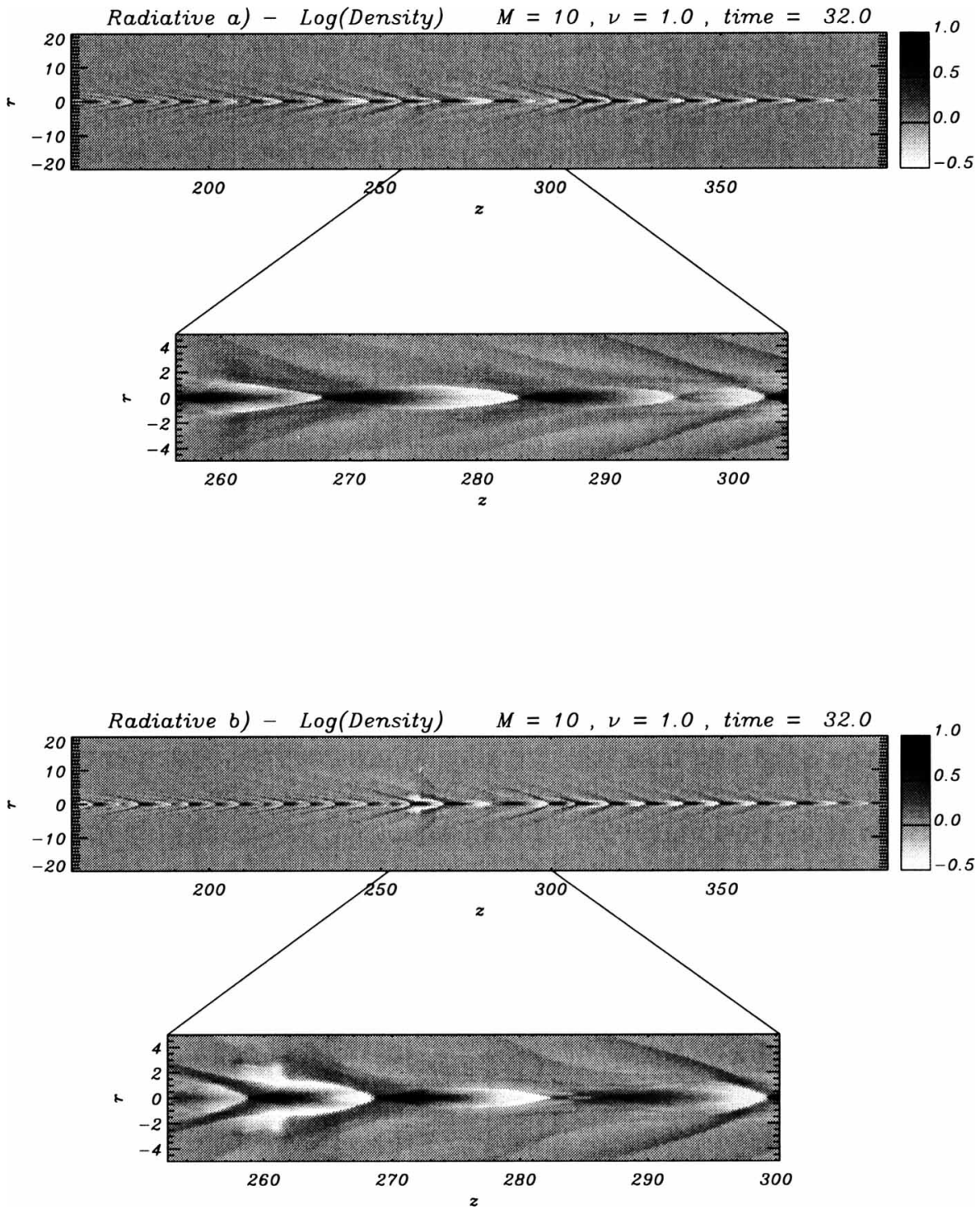

Figure 2. Gray-scale image of the logarithmic density distribution for for $\mathcal{H}=$ constant (upper panel) and for $\mathcal{H} \propto n_{\mathrm{H}}$ (lower panel). Figure refers to the evolution time $\tau=32$ 

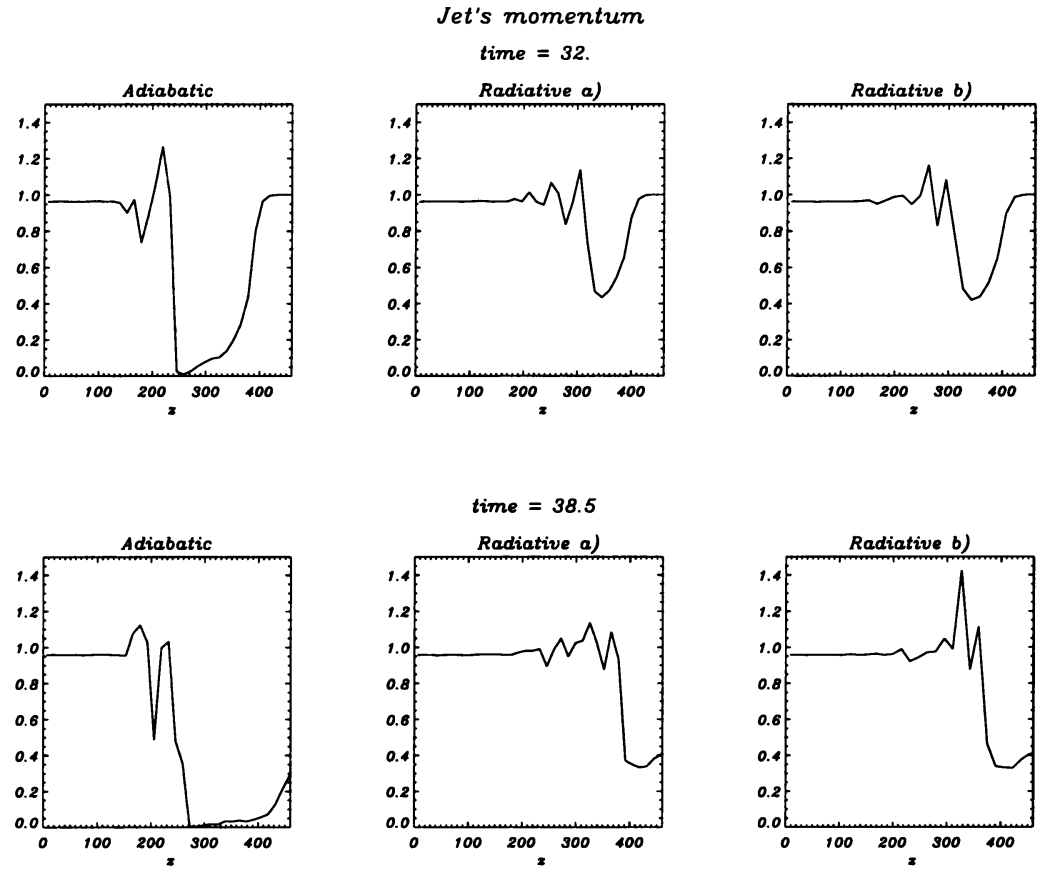

Figure 3. Average momentum carried by the jet against $z$. Upper panels for $\tau=32$, lower panels for $\tau=38.5$. From left to right: i) adiabatic limit, i) $\mathcal{H}=$ constant, $\mathcal{H} \propto n_{\mathrm{H}}$

regions upstream heating dominates cooling, but anyhow the mixing history of the jet is not ruled by these regions.

These calculations show that even a very crude assumption of the (unknown) heating term does not lead to severe limits on the results.

\section{References}

Birkinshaw, M., 1991, in Beams and Jets in Astrophysics, P.A. Hughes edt, Cambridge Univ. Press (Cambridge), chap. 6

Bodo G., Massaglia S., Ferrari A., Trussoni E. 1994, A\&A 283, 655

Bodo G., Massaglia S., Rossi P., Rosner R., Malagoli A., Ferrari A. 1995, A\&A 303, 281

Bührke T., Mundt R., Ray T.P. 1988, A\&A 200, 99

Colella P., Woodward P.R. 1984, J. Comp. Phys. 54, 174

Devine D.: 1997, in Low Mass Star Formation - from Infall to Outflow, Poster Proc. IAU Symp. No. 182, eds. F. Malbet \& A. Castets, Observ. de Grenoble, 1997, p. 95

Eislöffel J. and Mundt R. 1992, A\&A 263, 292

Hardee, P.E. and Stone J. 1997, ApJ in press.

Massaglia S., Trussoni E., Bodo G., Rossi P., Ferrari A. 1992, A\&A 260, 243

Massaglia S., Rossi P., Bodo G., Ferrari A. 1996, Ap. Lett. Comm. 34, 295

Micono M., Massaglia S., Bodo G., Rossi P., Ferrari A. 1997, A\&A in press.

Norman, M.L. and Hardee, P.E. 1988, ApJ, 334, 80 
Raga A.C., Cantó J., Binette L., Calvet N. 1990, ApJ 364, 601 Raymond, J.C. and Smith, B.R. 1977, ApJ. Suppl. 35, 419.

Reipurth B. and Heathcote S. 1992, A\&A 257, 693

Reipurth B., Raga A.C., Heathcote S.R. 1992, ApJ 392, 145

Rossi P., Bodo G., Massaglia S., Ferrari A. 1997, A\&A, in press

Stone J., Xu J., Hardee, P.E. 1997, ApJ in press. 